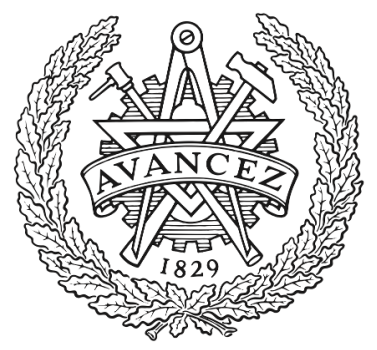

CHALMERS

UNIVERSITY OF TECHNOLOGY

\title{
Distributed Radar-aided Vehicle-to-Vehicle Communication
}

Downloaded from: https://research.chalmers.se, 2023-04-26 03:01 UTC

Citation for the original published paper (version of record):

Aydogdu, C., Liu, F., Masouros, C. et al (2020). Distributed Radar-aided Vehicle-to-Vehicle Communication. IEEE National Radar Conference - Proceedings.

http://dx.doi.org/10.1109/RadarConf2043947.2020.9266511

N.B. When citing this work, cite the original published paper. 


\title{
Distributed Radar-aided Vehicle-to-Vehicle Communication
}

\author{
Canan Aydogdu*, Fan Liu ${ }^{\dagger}$, Christos Masouros ${ }^{\dagger}$, Henk Wymeersch*, Mats Rydström ${ }^{\ddagger}$ \\ *Department of Electrical Engineering, Chalmers University of Technology, Gothenburg, Sweden \\ ${ }^{\dagger}$ Department of Electronic and Electrical Engineering, University College London, London, UK \\ ${ }^{\ddagger}$ Veoneer Research, Vårgårda, Sweden
}

\begin{abstract}
Establishing high-rate vehicle-to-vehicle (V2V) links with narrow beamwidth is challenging due to the varying network topology. A too narrow beam may miss the intended receiver, while a too broad beam leads to SNR loss. We propose to harness the high accuracy of radar detections to establish V2V links. In particular, we develop a distributed method where each vehicle associates local radar detections with GPS information communicated by nearby vehicles. The method relies on the transformation of relative to global coordinates, the definition of a suitable metric, and solving an optimal assignment problem. We demonstrate that the proposed approach avoids time-consuming channel estimation and provides high SNR, under the condition that reliable relative and absolute location information is present.
\end{abstract}

\section{INTRODUCTION}

Vehicles are expected to rely on wireless communication for several functionalities, including safety-related applications and traffic efficiency applications. Vehicle-to-vehicle (V2V) communication is currently standardized in ITS-G5, relying on WiFi-like protocols [1], [2]. Due to high mobility, intermittent connectivity, and the need for reliably and timely data transfer, as well as safety concerns, $\mathrm{V} 2 \mathrm{~V}$ communication is extremely challenging [3]. Multi-antenna techniques (MIMO) can help boost reliability, by providing a larger link budget through directional transmission [4]. However, this is only possible when channel state information (CSI) is known at the transmitter [5]. As obtaining such CSI entails additional overhead, directional transmissions are generally not considered and instead omnidirectional approaches are preferred. As a replacement for CSI, context information can be utilized, in particular location information [6]. For instance, the use of location information for directional beamforming was proposed in [7]. Both inband and out-of-band location information have been considered [8], [9]. Among the sensors that can provide location information, we mention radar and GPS, which are viewed as key enablers for $\mathrm{V} 2 \mathrm{~V}$ applications. Radar provides relative situational awareness, where backscattered signals are processed to construct a map [10]. Absolute situational awareness is provided by GPS, where signals acquired from satellites and ground stations are used to determine the 3D position of the vehicle on earth. Accuracy for automotive radar varies from 1 $\mathrm{cm}$ to $1 \mathrm{~m}$, and for GPS from $10 \mathrm{~cm}$ (for RTK GPS) to $10 \mathrm{~m}$ (for stand-alone GPS).

978-1-7281-8942-0/20/\$31.00 (c)2020 IEEE

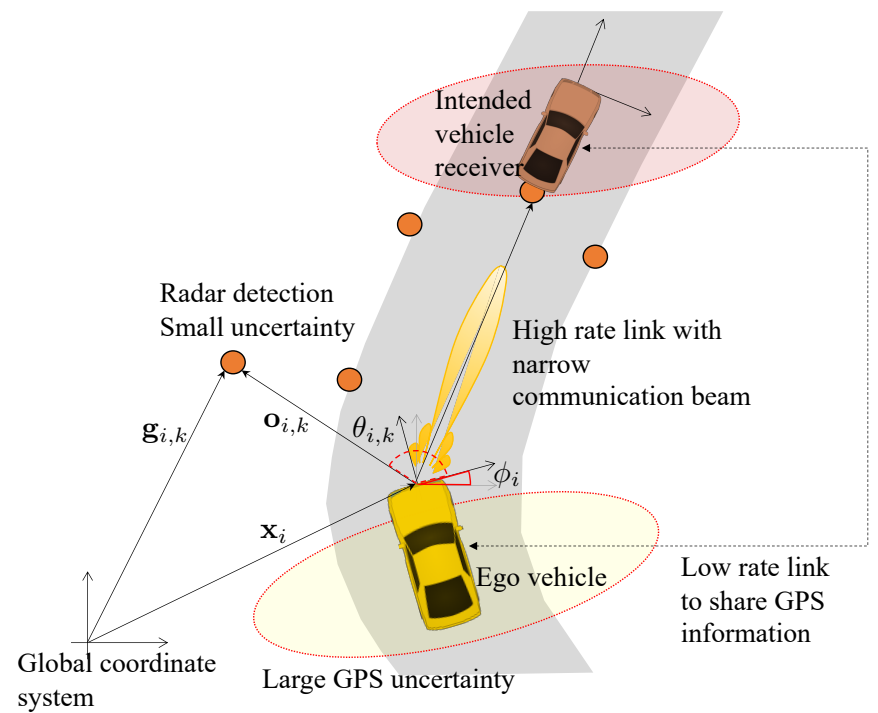

Figure 1. Scenario: the ego vehicle has unknown location and heading. It detects objects using a radar in its local frame of reference. Our goal is to determine which object corresponds to the intended receiver and then direct a narrow beam towards that receiver.

Recently, as terrestrial communication systems have started to operate in bands traditionally reserved for radar, there has been a growing interest in the convergence of communication and sensing [11], [12], where communication signals at high carrier frequencies are used for sensing the environment [13], [14] or where radar signals are used for communicating information [15], [16]. Automotive radar, which uses a large bandwidth at high carrier frequencies is an ideal technology for joint communication and sensing. It is then natural to identify and exploit synergies between the radar and communication functionalities.

In this paper, we propose radar-aided communication for V2V communication, develop a method for exploiting radar detections to steer highly directional communication signals. The method involves transforming radar detections to a global reference frame and then associating these transformed detections to vehicles. We analyze the impact of different accuracies of GPS and radar detections as well as different distance metrics between detected objects and vehicles. Our results indicate that radar-aware communication can increase link quality, but only when GPS is sufficiently accurate. 
Notation: We denote a multi-variate Gaussian density in variable $\mathbf{x}$ with mean $\boldsymbol{\mu}$ and covariance $\boldsymbol{\Sigma}$ by $\mathcal{N}(\mathbf{x} ; \boldsymbol{\mu}, \boldsymbol{\Sigma})$. We define the $2 \mathrm{D}$ rotation matrix

$$
\mathbf{R}(\phi)=\left[\begin{array}{cc}
\cos \phi & -\sin \phi \\
\sin \phi & \cos \phi
\end{array}\right] .
$$

Writing $[\mathbf{x}]_{k}$ returns the $k$-th entry of the vector $\mathbf{x}$.

\section{RELATED WORK}

The use of radar signals for improving communication has been studied in [17]-[22]. In [17], a BS equipped with a radar and communication sub-system (operating at different frequencies) is considered, where it is verified that the radar and communication channels share the same dominant paths. The paths detected by the radar are then used for directional data transmission. In [18], a similar problem to [17] is considered, but with radar and communication operating over the same band, but using separate waveforms. The radar recovers the angle of arrival (AoA) and angle of departure (AoD) of each path after which beamforming is designed towards those directions. In [19], 801.11ad communication at $60 \mathrm{GHz}$ is considered, where a BS router is configured to send and receive radar signals. This allows determination of vehicle range and velocity, within a certain beamwidth. The communication uses these estimates to beamform for maximum throughput. In [20], the radar at the BS is passive and listens to the vehicle radars. The azimuth power spectrum from the radar is then used for BS-to-vehicle communication. A method to address the lack of synchronization between vehicles and BS is proposed. In [21] an indoor mmWave communication system is developed, where an access point first senses the environment. Once the environment is mapped, communication towards a specific user in a certain location is performed based on predicted propagation paths (including reflected paths). Finally, in [22] a scenario similar to [17] is considered, but with a single joint communication and radar device. No downlink pilots are needed. A tracking algorithm predicts the vehicles' states and utilizes these predictions to perform power allocation for both communication and sensing. Among the above works, only [22] considers the unknown data association between radar detections and vehicles. None of the above works develop solution for the V2V scenario.

\section{SYSTEM MODEL}

\section{A. Problem Statement}

The system under consideration is visualized in Fig. 1, comprising vehicles and objects. Our focus is on the ego vehicle in yellow. We consider a scenario with multiple vehicles and objects distributed uniformly over an area observed at a snapshot in time, where motion models or road geometry are already subsumed in the available observation models from Section III-C.

Our aim is to offer a solution for the initial access problem, i.e., determining an optimal beam size and beam direction for high-rate data communication towards a specific set of vehicles, where local inherent radar measurements are used.
This radar-aided approach replaces the need for channel estimation, which becomes expensive and time-consuming in a dynamically changing vehicular environment. Moreover, with the introduction of ADAS and AD systems vehicles are expected to be equipped with radars that surround a vehicle. Hence, it becomes natural to use the already existing radar positioning capability in a distributed manner for initial access.

Our focus is not on delay- or safety-critical information exchange, since GPS is susceptible to jamming and spoofing and the initial access might be affected by attackers. However, authentication-type measures are possible over the formed high-rate beam. Hence, we are targeting either nonsafety-critical applications or safety-critical applications which are not delay-critical, such cooperative vehicular positioning through sensor raw-data sharing.

\section{B. Geometric Model}

We consider an environment with multiple vehicles with state in global coordinates of vehicle $i$ denoted by $\mathbf{s}_{i}=$ $\left[\mathbf{x}_{i}^{\top}, \mathbf{v}_{i}^{\top}, \phi_{i}\right]^{\top}$, where $\mathbf{x}_{i}$ is the $2 \mathrm{D}$ position, $\mathbf{v}_{i}$ is the $2 \mathrm{D}$ velocity, and $\phi_{i}$ is the heading (measured w.r.t. the front bumper, see Fig. 1). In addition to the vehicles, there are objects in the environment with states $\mathbf{g}_{k}$ (in global coordinates), comprising position and velocity. As object have no identifier, we will denote the state of an object seen by vehicle $i$ as $\mathbf{g}_{i, k}=\left[\mathbf{x}_{i, k}^{\top}, \mathbf{v}_{i, k}^{\top}\right]^{\top}$. Both vehicles and objects are modeled as point targets, with vehicle locations coinciding the radar equipment locations. Finally, we will consider a short transmission interval so that we can ignore mobility during the communication.

\section{Observation Model}

Each vehicle is equipped with a GPS device, which provides the density $p\left(\mathbf{s}_{i}\right)=\mathcal{N}\left(\mathbf{s}_{i} ; \overline{\mathbf{s}}_{i}, \mathbf{S}_{i}\right)$ in a global reference frame. Each vehicle also has a front-looking radar, which provides a set of $N_{i}$ detection objects within the field of view. Each object $k$ has an associated existence probability $\rho_{i, k} \in[0,1][23]$ and spatial density $p\left(\mathbf{o}_{i, k}^{\text {polar }} \mid \mathbf{s}_{i}\right)=\mathcal{N}\left(\mathbf{o}_{i, k}^{\text {polar }} ; \overline{\mathbf{o}}_{i, k}^{\text {polar }}, \mathbf{S}_{i, k}^{\text {polar }}\right)$ in the vehicle's local frame of reference. Here, $\mathbf{o}_{i, k}^{\text {polar }}=\left[r_{i, k}, \theta_{i, j}, v_{i, k}\right]^{\top}$ comprises distance $r_{i, k}=\left\|\mathbf{x}_{i}-\mathbf{x}_{i, k}\right\|$, AoA $\theta_{i k}$ (measured from the local $x$-axis), and radial velocity. We assume $\mathbf{S}_{i, k}^{\text {polar }}$ to be a diagonal matrix with measurement variances in range, AoA, and radial velocity.

\section{Communication Models}

Each vehicle is equipped with two separate communication systems. First of all a standard low rate system (e.g., 4G or ITS-G5 [2]) that provides GPS information from neighboring vehicles. The set of neighbors is denoted by $\mathcal{N}_{i}$. The availability of such a system is reasonable, as all modern vehicles are equipped with such communication transceivers to support a variety of services. While such a low-rate communication link for sharing GPS data introduces a latency, which results with outdated GPS data of other vehicles, this can be compensated by increasing the other vehicle's GPS covariance after 
reception, based on the received information and the latency between transmission and processing.

Secondly, each radar unit can serve as a directional communication transceiver [24], [25]. The direction of beamforming and the azimuth beamwidth can be controlled to achieve high SNR. The goal of vehicle $i$ is to determine $\left(\theta_{i j}^{\text {beam }}, W_{i j}^{\text {beam }}\right)$ in azimuth, in order to communicate with a neighboring vehicle $j \in \mathcal{N}_{i}$, without explicit channel estimation, in order to maximize the $\mathrm{SNR}^{1}$ [19]

$$
\begin{aligned}
& \mathrm{SNR}_{i j}= \\
& \begin{cases}\frac{P_{t}}{N_{0} W} \frac{4 \pi \lambda^{2}}{\left\|\mathbf{x}_{i}-\mathbf{x}_{j}\right\|^{2}} \frac{4 \pi^{2}}{W_{i j, \text { el }}^{\text {beam }} W_{i j}^{\text {beam }}} & \left|\theta_{i j}-\theta_{i j}^{\text {beam }}\right| \leq W_{i j}^{\text {beam }} \\
0 & \text { else, }\end{cases}
\end{aligned}
$$

where $\theta_{i j}$ is the direction of vehicle $j$ in the frame of reference of vehicle $i, W_{i j, \text { el }}^{\text {beam }}$ is a fixed beamwidth in elevation, $P_{t}$ is the $\mathrm{V} 2 \mathrm{~V}$ transmit power, $W$ is the $\mathrm{V} 2 \mathrm{~V}$ bandwidth, $\lambda$ is the wavelength at the $\mathrm{V} 2 \mathrm{~V}$ carrier frequency, and $N_{0}$ is the noise power spectral density.

\section{V2V LINK ESTABLISHMENT APPROACHES}

In this section, we propose two methods to determine $\left(\theta_{i j}^{\text {beam }}, W_{i j}^{\text {beam }}\right)$ based on location information: one based only on the communicated GPS data, and the second one based on the radar data.

\section{A. GPS-aided Communication}

In the frame of reference of vehicle $i$, the location of vehicle $j$ is $\mathbf{x}_{j}^{i}=\mathbf{R}\left(-\phi_{i}\right)\left(\mathbf{x}_{j}-\mathbf{x}_{i}\right)$. We thus find that

$$
\theta_{i j}=\arcsin \frac{\left[-\sin \phi_{i} \cos \phi_{i}\right.}{]^{\top}\left(\mathbf{x}_{j}-\mathbf{x}_{i}\right)} .
$$

In order to compute the distribution of $\theta_{i j}$, we use a particle approach. We first generate samples $\left[\mathbf{s}_{i}^{(p)} \mathbf{s}_{j}^{(p)}\right]_{p=1}^{N_{p}} \sim$ $p\left(\mathbf{s}_{i}\right) p\left(\mathbf{s}_{j}\right)$ and then evaluate $\theta_{i j}^{(p)}$ by computing (3) for each particle. Then,

$$
\theta_{i j}^{\text {beam }}=\mathbb{E}\left\{\theta_{i j}\right\} \approx \frac{1}{N_{p}} \sum_{p=1}^{N_{p}} \theta_{i j}^{(p)}
$$

and

$$
W_{i j}^{\text {beam }}=\alpha \sqrt{\frac{1}{N_{p}-1} \sum_{p=1}^{N_{p}}\left(\theta_{i j}^{(p)}-\theta_{i j}^{\text {beam }}\right)^{2}}
$$

where $\alpha>1$ is a constant to ensure that the beam covers the intended receiver.

\footnotetext{
${ }^{1}$ Note that we do evaluate report data rates, as we only deal with the initial access problem.
}

\section{B. Radar-aided Communication}

The proposed process involves three steps: (i) determining the distribution of $\mathbf{g}_{i, k}$ based on the radar measurements and vehicle $i$ 's GPS information; (ii) associating the vehicles to the objects, and (iii) computing the optimal beams.

Remark (On the use of velocity information). Since vehicle $i$ only has access to radial velocity measurements of the detected object, the distribution of $\mathbf{g}_{i, k}$ will have unbounded uncertainty in the velocity component. This is due to the lack of knowledge of the object's tangential velocity component. For that reason, we will drop the velocity from now on. The use of velocity information is possible when we consider explicit cooperation between multiple vehicles, as then the tangential component is also available and radial velocities between two mutually observable vehicles are identical. Such cooperation is out of the scope of the current work.

1) Coordinate Transformation: Our focus is on object $k$, detected by vehicle $i$. We first transform the object into Cartesian coordinates. If we denote the radial and AoA variances from $\mathbf{S}_{i, k}^{\text {polar }}$ by $\sigma_{r, i}^{2}$ and $\sigma_{b, i}^{2}$, respectively, then the object location in Cartesian coordinates $\mathbf{o}_{i, k}=\left[x_{i, k}^{i}, y_{i, k}^{i}\right]^{\top}$, in the vehicle frame of reference is $p\left(\mathbf{o}_{i, k} \mid \mathbf{s}_{i}\right)=\mathcal{N}\left(\mathbf{o}_{i, k} ; \overline{\mathbf{o}}_{i, k}, \mathbf{S}_{i, k}\right)$, where $\overline{\mathbf{o}}_{i, k}=\left[\bar{x}_{i, k}^{i}, \bar{y}_{i, k}^{i}\right]^{\top}$, in which

$$
\begin{aligned}
\bar{x}_{i, k}^{i} & =\bar{r}_{i, k} \cos \theta_{i, k} \\
\bar{y}_{i, k}^{i} & =\bar{r}_{i, k} \sin \theta_{i, k} \\
\mathbf{S}_{i, k} & =\mathbf{R}^{\top}\left(\theta_{i k}\right) \operatorname{diag}\left(\left[\sigma_{r, i}^{2}, \bar{r}_{i, k}^{2} \sigma_{b, i}^{2}\right]\right) \mathbf{R}\left(\theta_{i k}\right) .
\end{aligned}
$$

These relations follow from geometry. Then $\mathbf{g}_{i k}=$ $\mathbf{R}\left(\phi_{i}\right) \mathbf{o}_{i, k}+\mathbf{x}_{i}$, where all variables are random. The distribution of $\mathbf{g}_{i k}$ can be approximated as $\mathbf{g}_{i k} \sim \mathcal{N}\left(\mathbf{g}_{i, k} ; \overline{\mathbf{g}}_{i k}, \boldsymbol{\Gamma}_{i k}\right)$, where $\overline{\mathbf{g}}_{i k}=R\left(\bar{\phi}_{i}\right) \overline{\mathbf{o}}_{i, k}+\overline{\mathbf{x}}_{i, k}$ and

$$
\boldsymbol{\Gamma}_{i k}=\mathbf{F}_{i, k}\left[\begin{array}{cc}
\mathbf{S}_{i} & 0 \\
0 & \mathbf{S}_{i k}
\end{array}\right] \mathbf{F}_{i, k}^{\top}
$$

in which $\mathbf{F}_{i, k}$ is the Jacobian. Since $\nabla_{\phi_{i}} \mathbf{g}_{i k}=\mathbf{R}\left(-\phi_{i}\right) \mathbf{o}_{i, k}$, $\nabla_{\mathbf{x}_{i}} \mathbf{g}_{i k}=\mathbf{I}_{2}$ and $\nabla_{\mathbf{o}_{i, k}} \mathbf{g}_{i k}=\mathbf{R}\left(\phi_{i}\right)$,

$$
\mathbf{F}_{i, k}=\left[\begin{array}{lll}
\mathbf{I}_{2} & \mathbf{R}\left(-\bar{\phi}_{i}\right) \overline{\mathbf{o}}_{i, k} & \mathbf{R}\left(\bar{\phi}_{i}\right)
\end{array}\right]
$$

2) Data Association: We now associate the objects $k$ detected by vehicle $i$, transformed in the global coordinate system with the GPS data from the other vehicles $j$. In order to perform such an association, we introduce a similarity measure $D_{j k} \geq 0$ between object $k$ (detected by vehicle $i$ ) and vehicle $j \in \mathcal{N}_{i}$. These have densities $p\left(\mathbf{g}_{i, k}\right)$ and $p\left(\mathbf{s}_{j}\right)$, respectively. Recall that object also have a corresponding existence probability $\rho_{i, k}$. We propose to use the KullbackLeibler divergence (KLD), elaborated in the Appendix. Using 
this metric, we formulate an optimal assignment ${ }^{2}$ problem

$$
\begin{array}{ll}
\min _{\mathbf{X}} & \sum_{j \in \mathcal{N}_{i}} \sum_{k=1}^{N_{i}} x_{j k} D_{j k} \\
\text { s.t. } & x_{j k} \in\{0,1\} \\
& \sum_{j \in \mathcal{N}_{i}} x_{j k} \leq 1, \forall k \\
& \sum_{k=1}^{N_{i}} x_{j k}=1, \forall j .
\end{array}
$$

After solving the data association problem with the KuhnMunkres algorithm [27], we have determined $k^{*}(j)$, i.e., the best matching object to vehicle $j$.

3) Optimal Beams: The optimal beams are then easily found as

$$
\begin{aligned}
\theta_{i j}^{\text {beam }} & =\arcsin \left(\frac{\left[\overline{\mathbf{o}}_{i, k^{*}(j)}\right]_{2}}{\left\|\overline{\mathbf{o}}_{i, k^{*}(j)}\right\|^{2}}\right) \\
W_{i j}^{\text {beam }} & =\alpha \sqrt{\mathbf{f}_{i, k, j}^{\top} \mathbf{S}_{i, k^{*}(j)} \mathbf{f}_{i, k, j}}
\end{aligned}
$$

in which $\mathbf{f}_{i, k, j}$ is again a Jacobian, $\mathbf{f}_{i, k, j}=\nabla_{\mathbf{o}_{i k^{*}(j)}} \theta_{i j}$, so that

$$
\mathbf{f}_{i, k, j}^{\top} \mathbf{S}_{i, k^{*}(j)} \mathbf{f}_{i, k, j}=\frac{1}{\left\|\overline{\mathbf{o}}_{i, k^{*}(j)}\right\|^{2}} \mathbf{e}_{i, k, j}^{\top} \mathbf{S}_{i, k^{*}(j)} \mathbf{e}_{i, k, j},
$$

where $\mathbf{e}_{i, k, j}$ is a unit length vector, orthogonal to $\overline{\mathbf{o}}_{i, k^{*}(j)}$.

\section{REsults}

\section{A. Simulation Setting}

We consider an environment of size $50 \mathrm{~m}$ by $50 \mathrm{~m}$ in front of the ego vehicle. A total of 100 Monte Carlo simulations are run to obtain the results with the parameters summarized in Table I. The number of antenna elements are left unspecified, with the understanding that the implementation optimal beams in (4)-(5) and (12)-(13) depend on the array size, number of elements, and architecture (digital, analog or hybrid).

\section{B. Discussion}

We first evaluate the impact of GPS uncertainty. We set $\left[\mathbf{S}_{i}\right]_{1: 2,1: 2}=\sigma_{\mathrm{GPS}}^{2} \mathbf{I}_{2}$ and vary the value of $\sigma_{\mathrm{GPS}}$. The result in terms of average SNR (averaged over all links and over all Monte Carlo simulations) and average beamwidth are shown in Fig. 2. We compare three approaches: the GPS-aided method from Section IV-A, the radar-aided method from Section IV-B, with and without perfect data association. We observe that for low GPS variance, we achieve high average SNR, due to low beamwidth. With increasing GPS errors, beamwidth necessarily becomes larger, leading to SNR penalties. The radar-aided approach with perfect data association (DA) is not affected by the GPS variance, as it depends only on the radar performance, leading to high average SNR. Finally, with data association according to (11), high SNR is only achievable for low GPS variance. This is because in that case the objects, when transformed to global coordinates, remain well separated, leading to easy data association. When the

\footnotetext{
${ }^{2}$ Alternatively, soft data associations can be considered [26].
}

Table I

Default Simulation Parameters.

\begin{tabular}{ll}
\hline Parameter & Value \\
\hline V2V bandwidth $(W)$ & $100 \mathrm{MHz}$ \\
Carrier frequency $\left(f_{\mathrm{c}}\right)$ & $28 \mathrm{GHz}$ \\
V2V transmit power & $0.1 \mathrm{~mW}$ \\
Thermal noise temperature $T_{0}$ & $290 \mathrm{~K}$ \\
Receiver's noise figure & 1 \\
Range standard dev. $\sigma_{r}$ & $0.3 \mathrm{~m}$ \\
Bearing standard dev. $\sigma_{b}$ & $0.5^{\circ}$ \\
GPS heading standard dev. $\sigma_{\phi}$ & $0.57^{\circ}$ \\
Number of particles $N_{\mathrm{p}}$ & 500 \\
Beamwidth parameter $\alpha$ & 4 \\
Number of objects $N_{i}$ & 20 \\
Number of vehicles & 20 \\
Object existence prob. $\rho$ & 1.0 \\
\hline
\end{tabular}

GPS errors increase, data association errors occur (see below). These errors have limited impact on the beamwidth (since even under incorrect data association, beams can be narrow), but severely degrades the SNR. A drop of about $10 \mathrm{~dB}$ in average SNR is observed. We have also explored alternative distance metrics to the KLD, including Euclidean distance and Riemannian distance, but observed little performance difference.

The performance difference is explained in Fig. 3, showing the data association performance as a function of $\sigma_{\mathrm{GPS}}$. After about 1 meter standard deviation, about $10 \%$ of the associations are incorrect. After about 10 meter standard deviation, all the associations are incorrect. Hence, the selected beams will be narrow but point towards an incorrect directions. Note that these results are obtained for randomly distributed vehicles and objects, where the spatial separation in between might be lower compared to realistic road geometries. When vehicles are further apart than the GPS standard deviation, then the proposed method can deal with large GPS errors. Fig. 3 also includes an investigation of KLD performance for object existence probabilities $\left(\rho_{i, k}\right)$ of 0.5 , whereas the vehicle existence probabilities are 1. Although the data association error decreases marginally for lower object existence probabilities, the effect on beamwidth and SNR turns out to be negligible.

Finally, Fig. 4 shows the impact of the beamwidth parameter $\alpha$ on the average SNR and beamwidth. Unsurprisingly, larger $\alpha$ leads to broader beams, with the GPS-aided beams being about one order of magnitude wider than the radar-aided beams. The performance of the data association has no impact on the average beamwidth in this case. On the other hand, we see interesting behavior in the SNR: the GPS-aided approach generates too narrow beams for $\alpha<2$, leading to zero SNR. For increasing $\alpha$, beams cover the intended receiver, but SNR degrades as beams become wider. For the radar-aided approach, too small $\alpha$ leads to low SNR as the beams are unable to hit most of the intended receivers. Around $\alpha=2$ leads to optimal performance, with beams narrow enough to have high gains, but wide enough to illuminate the intended receiver. Increasing $\alpha$ beyond 2 leads to SNR reductions.

We have also investigated the performance sensitivity to the 

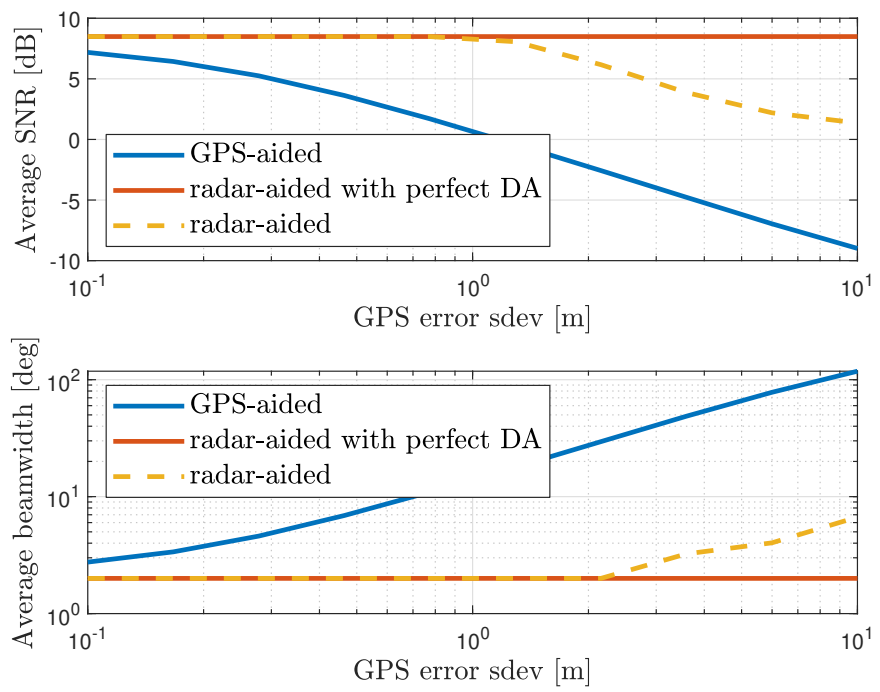

Figure 2. Performance comparison of GPS-aided and radar-aided V2V communication in terms of average SNR and average beamwidth, as a function of GPS standard deviation.

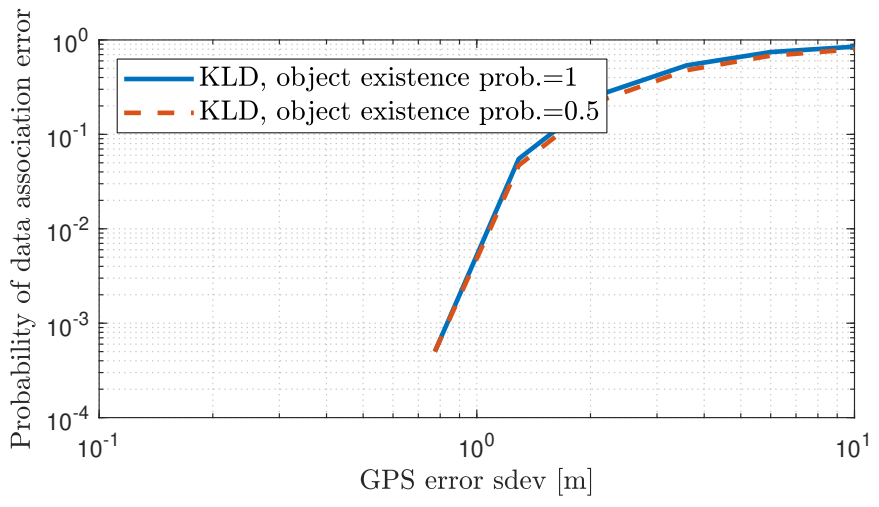

Figure 3. Probability of data association error for the scenario with 20 vehicles and 20 objects for changing GPS error standard deviation.

number of objects and the number of vehicles and found that the performance is reduced with increased number of vehicles.

\section{CONCLUSiOnS AND Future WORK}

We have proposed a method for radar-aided communication, which leverages the high accuracy of radar detections to form narrow beams towards intended receivers. To associate radar detections with receivers, we rely on GPS information from those receivers, communicated via an out-of-band technology. We adopt a simplified formulation by treating this problem in the context of localization rather than tracking, by focusing on a single snapshot. The method shows good performance when GPS errors are small with respect to the vehicle separation.

Possible extensions include more realistic models for radar performance, as well as extended object models; extending the model with velocities (to improve the data association) but this needs higher-level cooperation; consider soft data association (e.g., using belief propagation). In addition, a fair comparison
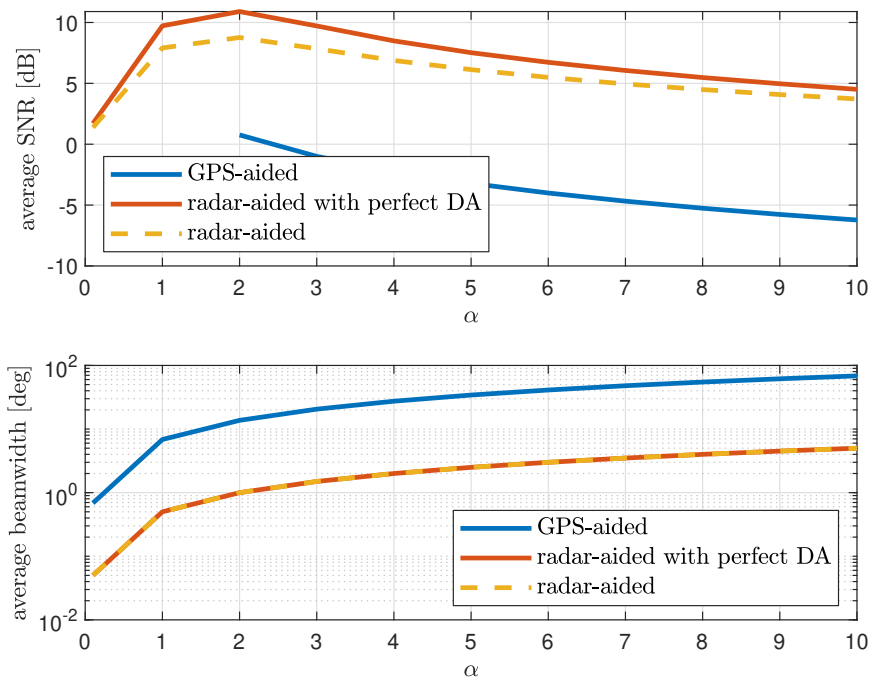

Figure 4. Performance comparison of GPS-aided and radar-aided V2V communication in terms of average SNR and average beamwidth, as a function of the beamwidth parameter $\alpha$.

with existing initial access methods should be conducted, accounting for practical array structures.

\section{ACKNOWLEDGMENTS}

This work is supported by Sweden's innovation agency (Vinnova) under grant 2018-01929 and the European Union's Horizon 2020 research and innovation programme under the Marie Skłodowska-Curie Grant Agreement No. 793345.

\section{APPENDIX}

\section{SIMILARITY MEASURE}

Given two Bernoulli set densities $f(\mathcal{X})$ and $g(\mathcal{Y})$, with existence probabilities $\rho_{f}$ and $\rho_{g}$ and spatial Gaussian densities $p_{f}(\mathbf{x})=\mathcal{N}\left(\mathbf{x} ; \boldsymbol{\mu}_{f}, \boldsymbol{\sigma}_{f}\right)$ and $p_{g}(\mathbf{y})=\mathcal{N}\left(\mathbf{y} ; \boldsymbol{\mu}_{f}, \boldsymbol{\sigma}_{f}\right)$. The KLD is then defined as

$$
\begin{aligned}
D(f \| g) & =\int f(\mathcal{X}) \log \frac{f(\mathcal{X})}{g(\mathcal{X})} \delta \mathcal{X} \\
& =f(\emptyset) \log \frac{f(\emptyset)}{g(\emptyset)}+\int f(\{\mathbf{x}\}) \log \frac{f(\{\mathbf{x}\})}{g(\{\mathbf{x}\})} \mathrm{d} \mathbf{x} \\
& =D\left(\rho_{f} \| \rho_{q}\right)+\rho_{f} D\left(p_{f} \| p_{g}\right) .
\end{aligned}
$$

where $D\left(\rho_{f} \| \rho_{q}\right)$ is the KLD between two Bernoulli distributions, i.e.,

$$
D\left(\rho_{f} \| \rho_{g}\right)=\left(1-\rho_{f}\right) \log \frac{\left(1-\rho_{f}\right)}{\left(1-\rho_{g}\right)}+\rho_{f} \log \frac{\rho_{f}}{\rho_{g}},
$$

and $D\left(p_{f} \| p_{g}\right)$ is the conventional KLD between two Gaussian densities, i.e.,

$$
\begin{aligned}
D\left(p_{f} \| p_{g}\right) & =\frac{1}{2} \operatorname{tr}\left(\boldsymbol{\Sigma}_{g}^{-1} \boldsymbol{\Sigma}_{f}\right)+\frac{1}{2} \log \left(\frac{\operatorname{det} \boldsymbol{\Sigma}_{g}}{\operatorname{det} \boldsymbol{\Sigma}_{f}}\right) \\
& +\frac{1}{2} \Delta^{\top} \boldsymbol{\Sigma}_{g}^{-1} \Delta-\operatorname{dim} / 2 .
\end{aligned}
$$

where $\Delta=\boldsymbol{\mu}_{g}-\boldsymbol{\mu}_{f}$ and dim is the dimension of the random variable. 
In case one of the variables is not a set, we can simply set the corresponding existence probability to 1 .

\section{REFERENCES}

[1] A. Festag, "Standards for vehicular communication - from IEEE 802.11 p to 5G," Elektrotechnik und Informationstechnik, vol. 132, no. 7, pp. 409-416, 2015.

[2] J. B. Kenney, "Dedicated short-range communications (DSRC) standards in the united states," Proceedings of the IEEE, vol. 99, no. 7, pp. 1162 1182, 2011.

[3] X. Yang, L. Liu, N. H. Vaidya, and F. Zhao, "A vehicle-to-vehicle communication protocol for cooperative collision warning," in IEEE International Conference on Mobile and Ubiquitous Systems: Networking and Services (MOBIQUITOUS), 2004, pp. 114-123.

[4] P. C. Eggers, T. W. Brown, K. Olesen, and G. F. Pedersen, "Assessment of capacity support and scattering in experimental high speed vehicle to vehicle MIMO links," in IEEE Vehicular Technology Conference (VTC), 2007, pp. 466-470.

[5] J. Wu and P. Fan, "A survey on high mobility wireless communications: Challenges, opportunities and solutions," IEEE Access, vol. 4, pp. 450476, 2016.

[6] R. Di Taranto, S. Muppirisetty, R. Raulefs, D. Slock, T. Svensson, and H. Wymeersch, "Location-aware communications for 5G networks: How location information can improve scalability, latency, and robustness of 5g," IEEE Signal Processing Magazine, vol. 31, no. 6, pp. 102-112, 2014.

[7] N. Garcia, H. Wymeersch, E. G. Ström, and D. Slock, "Locationaided mm-wave channel estimation for vehicular communication," in IEEE International Workshop on Signal Processing Advances in Wireless Communications (SPAWC), 2016.

[8] G. E. Garcia, N. Garcia, G. Seco-Granados, E. Karipidis, and H. Wymeersch, "Fast in-band position-aided beam selection in millimeter-wave MIMO," IEEE Access, vol. 7, pp. 142325-142338, 2019.

[9] G. C. Alexandropoulos, "Position aided beam alignment for millimeter wave backhaul systems with large phased arrays," in IEEE International Workshop on Computational Advances in Multi-Sensor Adaptive Processing (CAMSAP), 2017.

[10] S. M. Patole, M. Torlak, D. Wang, and M. Ali, "Automotive radars: A review of signal processing techniques," IEEE Signal Processing Magazine, vol. 34, no. 2, pp. 22-35, 2017.

[11] F. Liu, C. Masouros, A. Petropulu, H. Griffiths, and L. Hanzo, "Joint radar and communication design: Applications, state-of-the-art, and the road ahead," IEEE Transactions on Communications, vol. 68, no. 6, pp. $3834-3862,2020$

[12] B. Paul, A. R. Chiriyath, and D. W. Bliss, "Survey of RF communications and sensing convergence research," IEEE Access, vol. 5, pp. 252-270, 2016.

[13] J. Choi, V. Va, N. Gonzalez-Prelcic, R. Daniels, C. R. Bhat, and R. W. Heath, "Millimeter-wave vehicular communication to support massive automotive sensing," IEEE Communications Magazine, vol. 54, no. 12, pp. 160-167, 2016.
[14] P. Kumari, J. Choi, N. González-Prelcic, and R. W. Heath, "IEEE 802.11 ad-based radar: An approach to joint vehicular communication-radar system," IEEE Transactions on Vehicular Technology, vol. 67, no. 4, pp. 3012-3027, 2017.

[15] P. Barrenechea, F. Elferink, and J. Janssen, "FMCW radar with broadband communication capability," in European Radar Conference, 2007, pp. $130-133$.

[16] Y. L. Sit, C. Sturm, L. Reichardt, T. Zwick, and W. Wiesbeck, "The ofdm joint radar-communication system: An overview," in Proc. Int Conf. Advances in Satellite and Space Communications (SPACOMM), 2011, pp. 69-74.

[17] N. González-Prelcic, R. Méndez-Rial, and R. W. Heath, "Radar aided beam alignment in mmwave V2I communications supporting antenna diversity," in IEEE Information Theory and Applications Workshop (ITA), 2016

[18] Z. Chen, Z. Cao, X. He, Y. Jin, J. Li, and P. Chen, "DoA and DoD estimation and hybrid beamforming for radar-aided mmwave MIMO vehicular communication systems," Electronics, vol. 7, no. 3, p. 40, 2018.

[19] G. R. Muns, K. V. Mishra, C. B. Guerra, Y. C. Eldar, and K. R. Chowdhury, "Beam alignment and tracking for autonomous vehicular communication using IEEE 802.11 ad-based radar," in IEEE INFOCOM Workshops, 2019, pp. 535-540.

[20] A. Ali, N. González-Prelcic, and A. Ghosh, "Passive radar at the roadside unit to configure millimeter wave vehicle-to-infrastructure links," arXiv preprint arXiv:1910.10817, 2019.

[21] C. Jiao, Z. Zhang, C. Zhong, X. Chen, and Z. Feng, "Millimeter wave communication with active ambient perception," IEEE Transactions on Wireless Communications, vol. 18, no. 5, pp. 2751-2764, 2019.

[22] F. Liu, W. Yuan, C. Masouros, and J. Yuan, "Radar-assisted predictive beamforming for vehicular links: Communication served by sensing," arXiv preprint arXiv:2001.09306, 2020.

[23] B. Ristic, B.-T. Vo, B.-N. Vo, and A. Farina, "A tutorial on bernoulli filters: theory, implementation and applications," IEEE Transactions on Signal Processing, vol. 61, no. 13, pp. 3406-3430, 2013.

[24] A. Hassanien, M. G. Amin, E. Aboutanios, and B. Himed, "Dualfunction radar communication systems: A solution to the spectrum congestion problem," IEEE Signal Processing Magazine, vol. 36, no. 5, pp. 115-126, 2019.

[25] C. Aydogdu, M. F. Keskin, N. Garcia, H. Wymeersch, and D. W. Bliss, "RadChat: Spectrum sharing for automotive radar interference mitigation," IEEE Transactions on Intelligent Transportation Systems, 2019.

[26] Y. Bar-Shalom, F. Daum, and J. Huang, "The probabilistic data association filter," IEEE Control Systems Magazine, vol. 29, no. 6, pp. 82-100, 2009.

[27] J. Munkres, "Algorithms for the assignment and transportation problems," Journal of the Society for Industrial and Applied Mathematics, vol. 5 , no. 1, pp. 32-38, 1957. 